\title{
Influencia del tipo de escuela (especial o regular) y algunas variables socioeconómicas sobre el aprovechamiento escolar del alumnado con discapacidad
}

\author{
Influence of Type of School (Special or Regular) and some Socio-Economic Variables on \\ the Achievement of Students with Disabilities
}

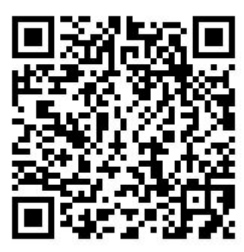

\author{
Ismael García-Cedillo' \\ Universidad Autónoma de San Luis Potosí \\ San Luis Potosí, México \\ ismaelgace@yahoo.com.mx \\ Silvia Romero-Contreras ${ }^{2}$ \\ Universidad Autónoma de San Luis Potosí \\ San Luis Potosí, México \\ romerosil@gmail.com
}

Recibido 13 de enero de 2015 • Corregido 10 de octubre de 2015 • Aceptado 17 de noviembre de 2015

Resumen. En México, el alumnado con discapacidad puede asistir a los centros de atención múltiple (CAM) o bien a algunas escuelas regulares para recibir su educación básica. El objetivo de este trabajo es identificar la influencia del tipo de escuela (CAM o escuela regular) y algunas variables socioeconómicas sobre el rendimiento académico estudiantil. Se trabajó con cinco pares de estudiantes con discapacidades similares de un preescolar regular y un CAM. Se aplicó la Batería de WoodcockMuñoz (BWM) para determinar el rendimiento académico, una entrevista a las madres y un protocolo de observación de aula. Se encontró que el alumnado de las escuelas regulares es más joven, proviene de hogares menos desfavorecidos y obtiene mejores puntajes en la BWM que el de CAM.

Palabrasclaves. Educación especial, educación básica, aprendizaje, integración educativa, discapacidad.

\footnotetext{
${ }^{1}$ Licenciatura, Maestría y Doctorado en Psicología Clínica por la Universidad Nacional Autónoma de México. Profesor-investigador de tiempo completo de la Universidad Autónoma de San Luis Potosí. Ha sido catedrático en distintas universidades del país, por ejemplo, la UNAM. Fue director del Proyecto Nacional de Integración Educativa, financiado por la Agencia Española de Cooperación Internacional y por la Secretaría de Educación Pública. Trabajó dos años como psicólogo clínico en el Latin American Health Institute, de Boston, Massachussets. Principal línea de investigación: la integración educativa/educación inclusiva, en la cual ha publicado varios libros y artículos científicos.

${ }^{2}$ Obtuvo la Licenciatura en Educación Especial en las áreas de Lenguaje, Aprendizaje y Audición en la Universidad de las Américas, la Maestría en Lingüística en la Escuela Nacional de Antropología e Historia y la Maestría y el Doctorado en Educación en la Universidad de Harvard. Sus líneas de investigación incluyen el desarrollo e implementación de modelos de intervención en las áreas de comunicación, lenguaje y lengua escrita con poblaciones de educación especial y regular; los procesos de educación inclusiva en la educación básica, y la formación docente en las áreas de lenguaje, lectura y educación inclusiva. Desde 2004, es profesora-investigadora de la Facultad de Psicología de la Universidad Autónoma de San Luis Potosí, donde enseña cursos en las áreas de metodología de la investigación y educación y coordina la Licenciatura en Psicopedagogía desde 2014. Es miembro del Sistema Nacional de Investigadores y líder del Cuerpo Académico Evaluación en Psicología y Educación.
} 
doi: http://dx.doi.org/10.15359/ree.20-1.1

URL: http://www.una.ac.cr/educare

CORREO: educare@una.cr

\begin{abstract}
In Mexico, students with disabilities can attend to multiple care centers (CAM, in its Spanish acronym) or to some regular schools to receive their basic education. The objective of this study is to identify the influence of the type of school (CAM or regular school) and some socio-economic variables on student academic performance. The work was carried out with five pairs of students with similar disabilities of a CAM and a regular preschool. The Woodcock-Muñoz battery (WMB) was applied to determine the academic performance, an interview with mothers and a classroom observation protocol. We found that the student body of regular schools is younger, descend from less disadvantaged homes and get better scores in the WMB than CAM.
\end{abstract}

Keywords. Special education, basic education, learning, educational integration, disability.

Tomasevsky (2002) plantea que el concepto de discapacidad ha sufrido cambios a lo largo de la historia. Cuando prevalecía un modelo tradicional o asistencial, los niños y niñas con discapacidad no tenían cabida en las escuelas; posteriormente, con el modelo médico, este alumnado tuvo acceso a las escuelas especiales. Con el modelo interactivo, se le aceptó en las escuelas regulares, lo que constituyó el proceso de integración educativa. Finalmente, con el modelo social se inició la inclusión, cuyo propósito fue abrir las escuelas regulares a toda la población infantil, con el objeto de ofrecerles una educación de calidad.

En México (y en muchos otros países) prevalece el modelo interactivo de la discapacidad, lo que implica que su aparición depende de la interacción entre las características del sujeto y de las condiciones del medio que lo rodea. La adopción de este modelo, como se podrá inferir de la conceptualización de Tomasevsky (2002), implica que en el país se desarrolla un proceso de integración educativa, más que de educación inclusiva.

Consecuente con este modelo interactivo de la discapacidad, para los niños y las niñas que la presentan (e incluso para quienes, sin tenerla, muestran un ritmo de aprendizaje por debajo del resto de sus compañeros y compañeras), existen tres opciones educativas:

a) Escuela regular sin apoyo de la USAER (Unidad de Servicios de Apoyo a la Educación Regular).

b) Escuela regular integradora (ERI), la cual cuenta con el apoyo de la USAER.

c) Escuela de educación especial, ahora llamada centro de atención múltiple (CAM) (Secretaría de Educación Pública, [SEP], 2006).

Aunque se desconoce su número, se cree que es poco el alumnado con discapacidad que estudia en las escuelas regulares sin apoyo, pues muchos maestros y maestras lo rechazan, entre otras razones porque consideran que no tienen la preparación suficiente para atenderlo; 
doi: http://dx.doi.org/10.15359/ree.20-1.1

URL: http://www.una.ac.cr/educare

CORREO: educare@una.cr

así, para la niñez con discapacidad se debe buscar su atención en una ERI o en un CAM. Las cifras proporcionadas por la última estadística de la SEP (2012) señalan que las escuelas regulares de educación básica (preescolar, primaria y secundaria) sin apoyo de educación especial (EE) en México son 167,651 (se desconoce en cuántas hay niñez integrada), en contraste con las 27,988 ERI. Se cuenta con 1,429 CAM (SEP, 2012). Según SEP (2012) es relativamente alto el número de niños y niñas que se atienden en las ERI o en los CAM $(654,782)$,; pero no se puede precisar cuántos se atienden por cada servicio.

En relación con la legislación, la Ley General de Educación, en su Artículo 41 señala que la educación especial debe atender al alumnado con discapacidad y a quienes presentan capacidades y aptitudes sobresalientes; agrega que se debe propiciar su integración a la escuela regular. Asimismo, plantea que "quienes no logren esa integración" podrán educarse en los CAM (Estados Unidos Mexicanos. Cámara de diputados del H. Congreso de la Unión, 2015, p. 18). Sin embargo, la imprecisión de dicha ley propicia que en los CAM se atienda al alumnado con discapacidades muy leves o incluso sin discapacidad (García, Romero, Motilla y Zapata, 2009; SEP, 2002). Generalmente, el alumnado llega al CAM canalizado por alguien del personal docente de educación regular o por algún o alguna integrante de las USAER o de los Centros de Recursos para la Integración Educativa (CRIE). Pese a lo anterior, la definición del tipo de escuela a la que debe asistir un niño o niña con discapacidad depende de sus padres y madres, quienes no siempre cuentan con la información más apropiada para tomar una decisión informada.

Es probable que la mayoría de hogares y algunos maestros o maestras ignoren que hay muchos estudios que muestran que la integración educativa favorece de manera sustantiva el desarrollo de la niñez con necesidades educativas especiales (NEE) con discapacidad o sin esta (Dyson Farrell, Polat, Hutcheson y Gallannaugh, 2004; Lupton, Thrupp y Brown, 2010; Ruijs, van der Veen y Peetsma, 2010; Samaniego, 2009). En contraste, casi no hay investigaciones publicadas sobre el aprovechamiento escolar (entendido en el presente trabajo como los aprendizajes alcanzados por estudiantes) del alumnado atendido en los CAM y menos aún estudios que comparen el aprovechamiento escolar de niñez con NEE similares y que estudie en CAM y ERI, lo cual probablemente obedece a la complejidad que representa su diseño.

Tomando en consideración lo anterior, la presente investigación tiene como objetivo comparar el nivel de aprovechamiento académico del alumnado, con NEE asociadas con alguna discapacidad, integrado en un preescolar regular apoyado por una USAER y estudiantes con características similares en un CAM. Se busca identificar si la integración educativa ha tenido un impacto significativo en los aprendizajes de los alumnos y alumnas integrados en preescolar regular en comparación con quienes asisten a una escuela especial, además de identificar algunas de las condiciones escolares y familiares en que estudian. 
doi: http://dx.doi.org/10.15359/ree.20-1.1

URL: http://www.una.ac.cr/educare

CORREO: educare@una.cr

\section{Referentes teóricos}

Durante muchos años, con la lógica de que con el acceso a la educación podían superarse las injusticias sociales y las diferencias de clase, México hizo grandes esfuerzos por aumentar la cobertura en la educación (Carrasco, 2008), y tuvo logros notables, pues de once y medio millones de estudiantes que atendía en 1970 pasó a casi treinta y cuatro millones en 2003 (SEP, 2013). Este logro se alcanzó, en parte, descuidando la calidad de la educación, asunto que últimamente ha cobrado gran relevancia, especialmente por los pobres resultados alcanzados por estudiantes mexicanos en las evaluaciones nacionales e internacionales sobre su rendimiento escolar (Organización para la Cooperación y el Desarrollo Económico [OECD], 2010; OREALC/Unesco (2008).

Hace años, la responsabilidad del bajo rendimiento escolar se tendía a adjudicar casi exclusivamente al estudiantado mismo. Con elavance de la investigación, se hallegadoa la noción de que el concepto de rendimiento escolar es multidimensional (Gutiérrez y Montañez, 2012), y se ha asociado con factores familiares como el nivel socioeconómico (Cervini, 2002; Gil, 2013; Hernández y González, 2011; Jiménez, 1988; Peña, 2002; Sentín, 2001) o con una combinación de indicadores de dicho nivel socioeconómico (Gil, 2013); con variables relacionadas con los recursos de la escuela como la infraestructura (Brunner y Elaqcua, 2004), con el profesorado (su formación, actualización, actitud frente a sus alumnos, habilidad para enseñar, expectativas sobre el aprendizaje de sus estudiantes, etc.), con la cultura (valoración de los aprendizajes en el grupo social del alumnado, valoración de la escolarización), las desigualdades familiares (Carrasco, 2008) y la adecuación del currículo, entre otros.

Si bien la investigación sobre los factores relacionados con el rendimiento escolar en las escuelas regulares es amplia, en México prácticamente no hay estudios publicados sobre la eficacia de las escuelas de educación especial en relación con la promoción de los aprendizajes de su alumnado y tampoco estudios que comparen la eficacia de estas escuelas con la de las escuelas regulares que atienden una población semejante.

El contexto dela educación especial enel país sedescribea continuación. Los principales servicios de educación especial, reorganizados en 1993 a partir del Acuerdo Nacional para la Modernización de la Educación Básica (Poder Ejecutivo Federal, 1992) funcionan de la siguiente manera:

1. Centros de atención múltiple (CAM), instituciones en las que se atiende al alumnado que presenta diferentes discapacidades, agrupado por edad (no por tipo de discapacidad); en teoría, se enseña el mismo currículo abordado por la escuela regular, con adaptaciones curriculares individuales. Se supone que solamente atienden a estudiantes que, por distintas circunstancias (fundamentalmente por tener discapacidades muy severas), no pueden estar integrados (SEP, 2002). Cuentan con un equipo de apoyo semejante al que conforma la USAER. 
doi: http://dx.doi.org/10.15359/ree.20-1.1

URL: http://www.una.ac.cr/educare

CORREO: educare@una.cr

2. El personal de USAER está constituido su director o directora y por una persona profesional de cada una de las siguientes áreas: psicología, comunicación, trabajo social; además, docentes de apoyo (uno o varios individuos). Las cuatro primeras funciones se ejercen de manera itinerante en un promedio de cinco escuelas regulares y el maestro o maestra de apoyo trabaja, por lo general, en una sola escuela. Una de las funciones principales de la USAER es apoyar la integración del estudiantado con NEE, mediante la realización de la evaluación psicopedagógica y la asesoría a la planta docente y a los padres de familia.

Cuando se inició la reorganización de los servicios de EE (que pretendía cambiar del modelo médico a un modelo más educativo), no se ofreció la capacitación suficiente al personal para que cambiara sus funciones, lo cual propició un cumplimiento discrecional, particularmente del personal de los CAM (García et al., 2009), por lo que se presentó lo que Guajardo (2010) denominó "desprofesionalización" de docentes de EE, aludiendo con este término al desconcierto que sufre el personal con respecto a sus roles y funciones.

Entrelas confusionesquepresentael personal de educación especial seencuentrala relacionada con las categorías que comprende el concepto de discapacidad. La Secretaría de Educación Pública cambió recientemente dichas categorías de discapacidad para ajustarlas a las propuestas por el Sistema Regional de Información Educativa de los Estudiantes con Discapacidad (Blanco, Mascardi y Narvarte, 2010), y son las siguientes: discapacidad física, intelectual, mental, múltiple, sensorial (auditiva y visual) y trastornos generalizados del desarrollo (Gobierno Federal. Comisión de política gubernamental en materia de derechos humanos, s. f.). Sin embargo, muy pocos profesionales de EE conocen esta clasificación, pues tanto los CAM como las USAER atienden a los alumnos y alumnas sin considerar si la situación que presentan corresponde o no a una discapacidad.

Como se dijo antes, hay pocas investigaciones que evalúan la efectividad de los CAM. Ezcurra (2004), por ejemplo, hizo un estudio en 20 CAM de ocho estados en el país, en los que aplicó cinco cuestionarios a personal directivo y docente, niñas y niños, familias y equipo de apoyo. Encontró prácticas de discriminación hacia la niñez con discapacidades severas; además, encontró que la organización de los CAM no favorece los aprendizajes del alumnado, pues organizan los grupos de acuerdo con su edad, lo que implica que en el mismo grupo estudian niños y niñas con distintas discapacidades y niveles de competencia curricular. Ponce, Pérez, López y Hernández (2006) realizaron una investigación en cuatro CAM del estado de Jalisco. Encontraron que los maestros y maestras dan preferencia al alumnado con discapacidades menos severas y que sus logros académicos son muy pobres. Los dos trabajos mencionados coinciden en que los CAM carecen de recursos y no cuentan con una normatividad definida.

Por su parte, López (s. f.), haciendo uso de su experiencia personal como profesional que trabaja en un CAM, señala que como en estas instituciones hay niños y niñas con discapacidades muy severas, difícilmente se puede seguir el currículo regular, razón por la cual el personal 
doi: http://dx.doi.org/10.15359/ree.20-1.1

URL: http://www.una.ac.cr/educare

CORREO: educare@una.cr

docente privilegia las áreas de desarrollo físico básico, la comunicación, el desarrollo de habilidades sociales y el autocuidado. La Red Internacional de Investigadores sobre Integración Educativa (2006) hizo una evaluación de la integración educativa en nueve estados del país, y como parte de esta evaluó el trabajo de cuatro CAM. Despúés de entrevistar a profesionales del CAM y a las autoridades encargadas de regularles, consideraron que los CAM constituyen "el servicio más olvidado del Programa Nacional para el Fortalecimiento de la Educación Especial y la Integración Educativa", no cuenta con propuesta ni con normatividad (p. 357).

En otra investigación, García et al. (2009) evaluaron el funcionamiento de cuatro CAM del estado de San Luis Potosí. Concluyeron que la reforma que se intentó realizar en estas instituciones resultó fallida porque los CAM se han organizado de distintas maneras, no de la forma en que se les indicó; no hay una normatividad definida; sus docentes enfrentan enormes retos para enseñar al alumnado, pues desconocen el currículo regular y tienen en sus grupos estudiantes con muy distintas discapacidades y niveles de competencia curricular; concluyen que un severo problema de los CAM es que permanecen, en general, invisibles dentro del sistema educativo mexicano.

\section{Método}

\section{Diseño}

El presente trabajo constituye un estudio descriptivo, de campo, intergrupal (se trabajó con dos muestras independientes) y transversal.

\section{Objetivos}

El objetivo general fue comparar el rendimiento académico de dos grupos de estudiantes con discapacidad que estudiaban en un Centro de Atención Múltiple (CAM) y en una escuela regular con apoyo de educación especial (ERI).

Se persiguieron los siguientes objetivos específicos:

1. Comparar las características socioeconómicas y otras variables de dos grupos, uno que atendía a un CAM y otro a una ERI.

2. Comparar las condiciones escolares y áulicas en que estudiaban dos grupos, uno que atendía a un CAM y otro a una ERI.

3. Comparar el rendimiento académico de dos grupos, uno que atendía a un CAM y otro a una ERI. 
doi: http://dx.doi.org/10.15359/ree.20-1.1

URL: http://www.una.ac.cr/educare

CORREO: educare@una.cr

\section{Muestra}

La muestra estuvo formada por dos grupos de cinco niños o niñas de edad preescolar, los cuales se describen a continuación:

Grupo I: estudiantes preescolares con necesidades educativas especiales asociadas a una discapacidad, que se integran a una ERI (se trabajó en tres escuelas).

Grupo II: estudiantes preescolares con necesidades educativas especiales asociadas a una discapacidad, que se atienden en CAM (una escuela).

En la tabla 1 se describen los dos grupos tal como quedaron conformados finalmente.

Tabla 1

Características de los sujetos por tipo de servicio: USAER-CAM

\begin{tabular}{cccll}
\hline Institución & Sexo & Edad (años/meses) & Grado escolar & Discapacidad \\
\hline ERI & F & $5: 9$ & $3^{\circ}$ preescolar & Discapacidad motora \\
& F & $6: 3$ & $3^{\circ}$ preescolar & Autismo \\
& M & $5: 6$ & $3^{\circ}$ preescolar & Debilidad visual/motora \\
& M & $6: 0$ & $2^{\circ}$ preescolar & Discapacidad intelectual/Síndrome de Down \\
& M & $5: 4$ & $2^{\circ}$ preescolar & Daño neurológico \\
\hline CAM & F & $7: 5$ & preescolar & Discapacidad intelectual de leve a moderada \\
& M & $7: 0$ & preescolar & Discapacidad intelectual de leve a moderada \\
& F & $6: 8$ & preescolar & Retraso psicomotor \\
& M & $7: 1$ & preescolar & Discapacidad intelectual/Síndrome de Down \\
& M & $7: 5$ & preescolar & Daño neurológico \\
\hline
\end{tabular}

Nota: Elaboración propia.

Se seleccionó a los sujetos de la investigación de manera no probabilística, por oportunidad; se realizó una revisión de sus expedientes gracias a la colaboración del equipo de USAER y del personal técnico y directivo del CAM. Se hizo todo lo posible para aparear los grupos de acuerdo con el tipo de discapacidad, el grado escolar y la edad (esto último no fue posible). Cada grupo estuvo integrado por cinco niños y niñas.

Además, participaron diez madres (correspondientes a los diez niños y niñas) y un padre, a quienes se realizaron diez entrevistas. Finalmente, se realizaron dos observaciones de aula, es decir, una para cada tipo de escuela (por cuestiones administrativas no se pudieron realizar observaciones en las otras escuelas regulares). 
doi: http://dx.doi.org/10.15359/ree.20-1.1

URL: http://www.una.ac.cr/educare

CORREO: educare@una.cr

\section{Instrumentos}

Se aplicaron tres instrumentos:

a) Prueba para evaluar la inteligencia y el aprovechamiento académico en estudiantes de edad preescolar, específicamente la Batería III Woodcok-Muñoz.

b) Entrevista semi estructurada, diseñada para la presente investigación. Se realizó a las madres de familia con el fin de conocer el ambiente familiar en el que se desenvuelven los niños y las niñas.

c) Formato de observación de clase, para indagar sobre el contexto de ambas instituciones.

A continuación se describe brevemente cada uno de los instrumentos:

- Batería III de Woodcock-Muñoz

La Batería IIIWoodcock-Muñoz fue traducida y adaptada para aplicarse a personas hablantes del español, además de que se calibraron los datos y se desarrollaron normas equivalentes a las de la Batería III de EEUU. Esta batería constituye la tercera edición. La calibración de los datos implicó la aplicación de la batería a 1,413 hablantes nativos del español (de quienes, 417 eran de Mexico). Para una descripción completa del proceso de calibración y adecuación de las normas de la batería, consultar a Schrank et al. (2005).

La Batería III Woodcock-Muñoz ofrece la posibilidad de evaluar el aprovechamiento escolar y las habilidades cognitivas desde varias dimensiones. En aprovechamiento escolar, es posible medir distintos aspectos del aprendizaje de la lectura, la escritura, el lenguaje oral y los conocimientos académicos (Woodcock y Muñoz-Sandoval, 1996). En este trabajo, se seleccionó una prueba de cada área de aprovechamiento, por considerarse que serían adecuadas para la población meta.

Se aplicaron las siguientes pruebas:

\section{Batería Woodcock-Muñoz III}

\section{Batería de habilidades cognitivas:}

- Comprensión verbal. Incluye cuatro subpruebas: Vocabulario sobre dibujos, sinónimos, antónimos y analogías verbales. La mediana de las confiabilidades de esta prueba es de 0.90 para sujetos de 5 a 19 años.

- Pareo visual (mediana de confiabilidad de 0.89).

- Palabras incompletas (mediana de confiabilidad de 0.77).

- Memoria para nombres (mediana de la confiabilidad de 0.88).

- Integración visual (mediana de confiabilidad de 0.80).

- Memoria para frases (mediana de confiabilidad de 0.89). 


\section{Batería de aprovechamiento académico:}

- Identificación de letras y palabras (mediana de la confiabilidad de 0.91)

- Ortografía (mediana de confiabilidad de 0.86).

- Problemas aplicados (mediana de confiabilidad de 0.92).

- Vocabulario sobre dibujos (mediana de confiabilidad de 0.77 )

\section{Compuestos}

\section{Compuestos de rendimiento cognitivo}

- Habilidad intelectual general

- Habilidad verbal

\section{Compuestos de rendimiento académico}

- Aprovechamiento breve

- Conocimiento académico

- Entrevista

Las entrevistas a las madres se realizaron antes de la aplicación de la prueba WoodcokMuñoz, para que ellas estuvieran enteradas y pudieran dar su consentimiento para la realización de la investigación. La entrevista fue elaborada a partir de la información que se pretendía recoger, en este caso sobre las condiciones de vida de las familias involucradas en la investigación: las características socioeconómicas, físicas y, en general, el ambiente familiar. Además, se puso énfasis en conocer a qué tipo de información tenían acceso los alumnos y alumnas en sus casas y también el nivel de satisfacción y expectativas escolares. La duración de la entrevista fue aproximadamente de media hora, en ocasiones se realizaron preguntas de seguimiento para ampliar la información y evitar dudas y confusiones.

- Formato de observación

El formato de observación utilizado en esta investigación fue tomado de la investigación sobre integración educativa realizada por García et al. (2003). Para esta investigación se realizaron algunas adecuaciones al formato original para registrar la actividad observada y algunas características de la escuela y del aula. 
doi: http://dx.doi.org/10.15359/ree.20-1.1

URL: http://www.una.ac.cr/educare

CORREO: educare@una.cr

\section{Resultados}

\section{Resultados de las entrevistas a madres de niños de CAM}

Todas las entrevistadas eran las madres de los niños y las niñas, quienes mostraron buena disposición. Las entrevistas se realizaron en un espacio asignado y tuvieron una duración aproximada de 30 minutos. Se realizaron en total cinco entrevistas. Se encontró que todas las familias viven en la periferia de la ciudad (San Luis Potosí, México) o en comunidades cercanas, por lo cual tienen que recorrer distancias considerables (de más de una hora) y lo hacen en transporte público. Tres de las madres conocen el diagnostico clínico de su hijo o hija, aunque sus respuestas resultaron confusas y usaron un lenguaje impreciso para describir su situación. Solo uno de los niños toma medicamento, recetado por un psiquiatra.

Tres de las familias han vivido la situación de su hijo o hija sin significarla de manera especial ("no he batallado con él, se comporta como cualquier otro niño"), mientras que dos de las entrevistadas argumentan que ha sido difícil sobrellevar la situación ("no esperábamos la discapacidad del niño"). Cuatro de las familias son de tipo nuclear, solo una de las familias es extensa, el alumnado participante tiene en promedio dos hermanos o hermanas y viven con ambos progenitores.

Todas las madres entrevistadas son amas de casa, por tanto, el único sustento económico es el proporcionado por el padre; las familias pertenecen a un nivel socioeconómico medio bajo a bajo; la ocupación de los padres se relaciona con oficios como albañilería, comercio y operarios de transportes.

Cuatro familias viven en casa propia y una en casa rentada, cuentan con servicios básicos de agua, electricidad, drenaje y tienen teléfono, no cuentan con internet. Los niños y niñas comparten la habitación con alguien más, tres de ellos con alguno de sus hermanos o hermanas y dos con sus madres; duermen en promedio diez horas. Acostumbran comer algunos alimentos antes de ir a la escuela tales como leche, huevo, frijoles, cereal, solo uno de los niños no consume alimentos en la mañana.

En cuanto a la tecnología disponible y material didáctico en casa, nadie cuenta con equipo de cómputo ni sabe utilizar la computadora, solo dos familias cuentan con algún libro infantil (no más de 2 o 3) y únicamente una familia se preocupa por comprar juguetes educativos.

Los niños y niñas interactúan con muy pocos pares de su edad fuera de la escuela (solo una de las madres mencionó que su hijo jugaba con amiguitos y primos), pero sí juegan con los hermanos y con sus progenitores. Cuatro acostumbran ver televisión un promedio de cuatro horas al día, y acostumbran salir de paseo al parque, centros comerciales y plazas públicas o a visitar a familiares. 
doi: http://dx.doi.org/10.15359/ree.20-1.1

URL: http://www.una.ac.cr/educare

CORREO: educare@una.cr

En cuanto al área académica, la mayoría ha asistido varios años al CAM; no estuvieron en guardería, por lo mismo no han vivido experiencias escolares de rechazo; no reciben apoyo extra en alguna otra institución pública o privada. Dos fueron canalizados a esta institución por la planta docente de una escuela regular y otro por un médico.

En relación con las expectativas de las madres, dos de ellas comentan que les gustaría que sus hijos entraran a una escuela primaria regular; una madre cree que su hijo puede terminar una licenciatura, tres de ellas piensan que sus hijos o hijas llegarán a terminar la primaria, pero dentro del CAM.

En el área social, las mamás reportan que sus hijos tienen pocos amigos y amigas en casa, tres de ellos no conviven regularmente con sus compañeros, y dos de ellos sí, pero con menos de cinco compañeros. En el hogar, la interacción con otros niños y niñas de su edad es escasa, en promedio menos de cinco niños de su edad (vecinos o primos), sin contar a los hermanos. Tres de las mamás comentan que la conducta de sus hijos ha mejorado desde que asisten al CAM, y los describen como amigables y a veces agresivos.

En cuanto al nivel de satisfacción de las madres con respecto al aprendizaje de sus hijos e hijas, cuatro de ellas comentan estar satisfechas y una muy satisfecha. Todas las mamás han notado cambios en sus hijos e hijas, la mayoría favorables, tales como mejoría en el lenguaje, mayores conocimientos y mejor conducta, y algunos cambios desfavorables como dificultades escolares. En general, las madres califican el desempeño del personal docente y de especialistas de la institución como bueno. Ninguna de las madres ha recibido información sobre la integración educativa/educación inclusiva: esta terminología les resultó desconocida.

\section{Resultados de las entrevistas a madres de niños en las ERI}

Se entrevistó en total a cuatro mamás y a una pareja de papá y mamá. Al preguntar sobre la dificultad del niño o niña, las madres describieron su condición clínica (parálisis cerebral, síndrome de Down, etc.); todos los niños y niñas cuentan con un diagnóstico clínico y las madres tienen un conocimiento amplio de su situación. Cuatro niños toman medicamento regularmente. Los niños reciben apoyo extraescolar del Centro de Rehabilitación y Educación Especial o del Centro de Apoyo Psicopedagógico para la Educación Preescolar (tratamiento neurológico, de lenguaje o de aprendizaje). Cuatro entrevistados comentan que en la familia han vivido la situación de sus hijos o hijas como difícil, debido a que presentan complicaciones médicas y desde temprana edad han asistido a muchas terapias.

Tres de las familias son de tipo nuclear (papá, mamá y hermanos y hermanas), y dos extensas (por la presencia de abuelos). Son familias de un nivel socioeconómico medio bajo a medio alto, las madres tienen estudios de preparatoria y una terminó la licenciatura; tres de las madres son amas de casa, una es estilista y otra es empleada de almacén. 
doi: http://dx.doi.org/10.15359/ree.20-1.1

URL: http://www.una.ac.cr/educare

CORREO: educare@una.cr

Con excepción de un caso, la madre es quien pasa el mayor tiempo con el hijo o hija, los padres pasan pocas horas ya que la mayor parte del tiempo trabajan. Cuando ambos progenitores se ausentan, generalmente es una de las abuelas quien cuida del menor.

Cuatro de las familias viven en casa propia, y una en una casa rentada, todas cuentan con servicios básicos de drenaje, luz, agua, cuatro de ellas tienen teléfono y solo una cuenta con internet. Tres de los niños comparten la habitación con alguien más, y dos de ellos comparten la cama con sus progenitores o abuelita. Duermen en promedio entre 9 y 10 horas al día, todos acostumbran consumir algún alimento ligero antes de ir a la escuela (licuado, huevo, quesadillas, pan, etc.).

A cuatro niños los trasladan a la escuela en auto propio, tres de las familias cuentan con computadora en casa y también tres de los niños saben manejarla; en promedio, las familias dicen tener cinco libros infantiles en casa, y seis juguetes didácticos.

El principal pasatiempo de cuatro de los niños es el juego, el tiempo que dedican a jugar varía entre media hora hasta tres horas. Los niños juegan solos o con sus hermanos o primos. Los niños y niñas acostumbran ver televisión entre una y dos horas al día, realizan paseos familiares en promedio una vez cada 15 días y los lugares más mencionados de visita fueron: parques, centros comerciales, plazas públicas y visitas familiares.

Dos de los niños fueron rechazados en otros preescolares antes de inscribirse al actual; cuatro de las madres comentan que inscribirán a sus hijos en una primaria regular, solo una comentó que inscribirá a su hijo en una escuela de educación especial. En cuanto a las expectativas de las madres, tres de ellas esperan que sus hijos terminen una licenciatura, y dos de ellas que terminen la primaria.

En general, las madres comentan que han notado un cambio positivo en la manera en que sus hijos se relacionan con los demás niños, conviven con un número alto de compañeros, los describen como niños sociables y en ocasiones agresivos. Ninguno de los niños interactúa frecuentemente con pequeños de su edad en casa, únicamente con los hermanos y esporádicamente con primos.

Todas las mamás están satisfechas con el aprendizaje logrado por sus hijos e hijas, han notado cambios favorables en cuanto a la socialización, lenguaje y conocimientos. Dos madres comentaron algunos cambios desfavorables, sobre todo en la conducta (agresividad, berrinches). Cuatro de las mamás comentan estar satisfechas con la organización de la escuela, y no les gustaría que hubiera cambios, solo una madre comenta que le gustaría que no hubiera tanta rotación de maestras. En general, las mamás calificaron como bueno o muy bueno el desempeño del personal docente, el desempeño del personal de USAER y de la institución educativa. Ninguna de las mamás cambiaría a sus hijos o hijas a un CAM, por la creencia generalizada de que los avances son menores, ya que tienden a imitar a los demás niños y niñas. Tres de las mamás han recibido información sobre integración educativa/educación inclusiva, principalmente de la USAER, y tienen opiniones positivas acerca de esta modalidad; comentan que ha sido un gran apoyo para ellas, es un derecho para los niños, y es una manera de integrarles a la sociedad. 


\section{A continuación, en el tabla 2, se presenta un resumen de las entrevistas a las madres.}

Tabla 2

Resumen de respuestas de las madres de familia a la entrevista

\begin{tabular}{|c|c|c|c|c|}
\hline \multicolumn{5}{|c|}{ Tipo de servicio } \\
\hline Ítem & frec & CAM & frec & USAER \\
\hline \multirow[t]{2}{*}{ Realizada a } & \multirow[t]{2}{*}{5} & \multirow[t]{2}{*}{ La madre } & 4 & La madre \\
\hline & & & 1 & Madre y padre \\
\hline \multirow[t]{2}{*}{ Medio de transporte } & \multirow[t]{2}{*}{5} & \multirow[t]{2}{*}{ Transporte público } & 4 & Auto propio \\
\hline & & & 1 & Transporte público \\
\hline ¿Conocen el diagnóstico? & 3 & Sí, vagamente & 5 & Sí, con precisión \\
\hline ¿Reciben apoyo extra-escolar? & 5 & No & 5 & $\begin{array}{l}\text { Sí, en instituciones públicas } \\
\text { (CREE, CAPEP) }\end{array}$ \\
\hline \multirow[t]{2}{*}{ Tipo de familia } & 4 & $\begin{array}{l}\text { Nuclear-padre-madre y dos o } \\
\text { tres hijos }\end{array}$ & 3 & Nuclear-madre, padre e hijos. \\
\hline & 1 & Extensa & 2 & Extensas \\
\hline \multirow[t]{3}{*}{ Ocupación de la madre } & \multirow[t]{3}{*}{5} & \multirow[t]{3}{*}{ Amas de casa } & 3 & Amas de casa \\
\hline & & & 1 & Estilista \\
\hline & & & 1 & Empleada de almacén \\
\hline \multirow[t]{2}{*}{ Escolaridad de la madre } & & \multirow[t]{2}{*}{ ND } & 4 & Preparatoria \\
\hline & & & 1 & Licenciatura terminada \\
\hline Ocupación del padre & 5 & $\begin{array}{l}\text { Oficios con calificación } \\
\text { intermedia (albañil, } \\
\text { comerciante, operario de } \\
\text { transportes) }\end{array}$ & 5 & $\begin{array}{l}3 \text { amas de casa } \\
1 \text { estilista } \\
1 \text { empleada de almacén }\end{array}$ \\
\hline Nivel socioeconómico & 5 & Medio-bajo & 5 & Entre medio-bajo y medio-alto \\
\hline \multirow[t]{2}{*}{ Vivienda } & 4 & Propia & 4 & Propia \\
\hline & 1 & Rentada & 1 & Rentada \\
\hline
\end{tabular}


doi: http://dx.doi.org/10.15359/ree.20-1.1

URL: http://www.una.ac.cr/educare

CORREO: educare@una.cr

\begin{tabular}{|c|c|c|c|c|}
\hline \multicolumn{5}{|c|}{ Tipo de servicio } \\
\hline Ítem & frec & CAM & frec & USAER \\
\hline \multirow{2}{*}{$\begin{array}{l}\text { Materiales didácticos-libros } \\
\text { en casa }\end{array}$} & 2 & Libros infantiles (2 o 3) & 5 & Libros infantiles y juegos didácticos \\
\hline & 1 & Juegos didácticos & & \\
\hline \multirow[t]{2}{*}{$\begin{array}{l}\text { ¿Amigos del niño fuera de la } \\
\text { escuela? }\end{array}$} & 4 & $\begin{array}{l}\text { No, juegan solo con } \\
\text { progenitores y hermanos }\end{array}$ & 5 & $\begin{array}{l}\text { No, solo con hermanos y } \\
\text { primos }\end{array}$ \\
\hline & 1 & Sí, juega con amigos y primos & & \\
\hline Exposición a TV & 4 & 4 horas diarias & 5 & Entre 1 y 2 horas diarias \\
\hline Escolaridad previa & & 4 Siempre en CAM & 2 & $\begin{array}{l}\text { Rechazados de otros } \\
\text { preescolares }\end{array}$ \\
\hline \multirow[t]{4}{*}{ Expectativas académicas } & 2 & Que ingrese a una primaria regular & 4 & Que ingrese a primaria regular \\
\hline & & & 1 & $\begin{array}{l}\text { Que ingrese a escuela especial- } \\
\text { Instituto para ciegos }\end{array}$ \\
\hline & 1 & $\begin{array}{l}\text { Cree que su hijo puede } \\
\text { terminar una licenciatura }\end{array}$ & 3 & Que termine la licenciatura. \\
\hline & 1 & $\begin{array}{l}\text { Que terminen la primaria } \\
\text { dentro del CAM }\end{array}$ & 2 & Que termine la primaria \\
\hline Avances en la escuela actual & & $\begin{array}{l}\text { En general, reportan avances } \\
\text { en lenguaje, conocimientos, } \\
\text { conducta y algunas } \\
\text { dificultades escolares }\end{array}$ & & $\begin{array}{l}\text { Cambio positivo en la } \\
\text { socialización, ocasionalmente } \\
\text { agresivos }\end{array}$ \\
\hline \multirow{3}{*}{$\begin{array}{l}\text { Satisfacción con la escuela } \\
\text { actual }\end{array}$} & 4 & Satisfechas & 4 & Satisfechas \\
\hline & & & 1 & $\begin{array}{l}\text { Regular, "que haya menos } \\
\text { rotación de maestras" }\end{array}$ \\
\hline & 1 & Muy satisfecha & 5 & $\begin{array}{l}\text { No cambiarían a su hijo a un } \\
\text { CAM }\end{array}$ \\
\hline $\begin{array}{l}\text { ¿Tiene información sobre } \\
\text { integración educativa? }\end{array}$ & 5 & $\begin{array}{l}\text { No, no han recibido } \\
\text { información, el término les } \\
\text { resultó desconocido }\end{array}$ & 3 & $\begin{array}{l}\text { Sí, de parte de la USAER-tienen } \\
\text { opinión positiva de cómo } \\
\text { funciona la integración educativa }\end{array}$ \\
\hline
\end{tabular}

Nota: Elaboración propia. 


\section{Resultados de las observaciones}

La observaciones de aula se realizaron todas al inicio de la jornada académica, es decir, a las nueve de la mañana, y tuvieron una duración entre 30 y 40 minutos; se observó una actividad específica, a los dos grupos se les aviso previamente el día y la hora en que se realizaría la observación y en las dos aulas se aplicó el mismo formato. A continuación se describe la información más relevante obtenida de la observación en clase

\section{Aula de CAM}

En cuanto a las condiciones físicas del aula, el nivel de ruido, temperatura, tamaño y ventilación son adecuadas; el salón no está muy limpio, pues hay basura en el piso. Las paredes del aula lucen casi desnudas, únicamente tienen algún material elaborado por el maestro o maestra y muy pocos dibujos de estudiantes, quienes están sentados en mesas para cuatro, con asientos no fijos, acomodados en herradura. El aula cuenta con un escritorio para el personal docente, librero y archivero un tanto maltratados y viejos. El material didáctico que existe en el aula es adecuado para el nivel de sus estudiantes, pero es escaso y también se observa maltratado.

La actividad observada consiste en trabajar la expresión oral y realizar un pequeño masaje en la cara, cerca de la boca. Durante el momento de la observación hay 16 alumnos y alumnas y algunas madres apoyando la actividad, además de una estudiante normalista, quien realiza sus prácticas en dicha institución.

La maestra encargada del grupo cuenta con una planeación mensual por escrito, comenta que cada mes realiza un proyecto.

En cuanto a la metodología utilizada por la maestra, se observa que explica previamente de qué va a tratar la actividad, pero no se cerciora de que sus estudiantes entiendan, la manera de motivarles es mencionando sus logros y se enfoca únicamente en un canal de entrada, el auditivo. Durante la actividad, los alumnos y alumnas se muestran participativos al inicio, pero van perdiendo interés, hasta que al final muestran aburrimiento y se distraen fácilmente.

Los materiales utilizados son concretos. Durante la actividad se presentan algunos problemas de indisciplina, ya que algunos alumnos se levantan de su asiento, a lo cual la maestra responde regañando a los responsables frente al grupo. Las dos principales fortalezas de la maestra son: a) buen uso del lenguaje oral, el cual es claro y entendible, apropiado para el nivel de los niños y las niñas; b) tratar afectivamente y con respeto a los alumnos y alumnas. Las dos principales dificultades son: a) propiciar la dependencia, ya que se observa una tendencia repetitiva a realizar el trabajo de los niños y al brindarles apoyo excesivo; b) toda la clase realiza la misma actividad, y se exige el mismo resultado, al mismo tiempo. La relación entre la maestra y sus estudiantes es buena, caracterizada por respeto, afectividad y aceptación, mientras que la relación entre los niños se caracteriza por respeto, aunque en ocasiones muestran indiferencia e individualismo. 
doi: http://dx.doi.org/10.15359/ree.20-1.1

URL: http://www.una.ac.cr/educare

CORREO: educare@una.cr

\section{Aula de la ERI}

Se trata de un grupo de preescolar, de tercer grado. Durante la observación hay 22 alumnos y alumnas, una con NEE asociada a discapacidad (daño neurológico).

El aula es amplia, con muy buena iluminación, temperatura adecuada, nivel de ruido bajo (no impide la realización de la actividad). Además, hay buena ventilación y limpieza. Las paredes del aula están decoradas con material elaborado por los alumnos, las alumnas y la maestra, además de láminas y mapas.

El mobiliario consta de mesas para dos, con asientos no fijos, acomodados en forma de herradura; hay además un escritorio, librero, archivero y gaveta, todos en buenas condiciones. Se cuenta con un espacio para el material didáctico, el cual es numeroso y variado.

La actividad observada está coordinada por la maestra de apoyo; consiste en contar un cuento utilizando diferentes materiales (muñecos, peluca, platos y cucharas). El cuento es "Ricitos de oro y los tres ositos".

La maestra de apoyo cuenta con una planeación por escrito. Durante la actividad se promueve la participación de la alumna con NEE, pues se le solicita que interprete a Ricitos de oro; el uso del tiempo es adecuado, no hay tiempos muertos. Los alumnos y alumnas se mantienen interesados, atentos y participativos, hay mínimas dificultades de conducta, solo algunos alumnos inquietos que se controlan adecuadamente.

En cuanto a la metodología de la maestra, consiste en formular preguntas para conocer el grado de conocimientos, elogiar las participaciones, se relaciona la información con actividades cotidianas. También se observa que la maestra toma en cuenta los diferentes estilos de aprendizaje, al utilizar los canales visual, auditivo y kinésico.

Durante el desarrollo de la actividad, la alumna con NEE hace lo mismo que el resto de sus compañeros, la maestra le presta más atención a ella, le da ayuda física y le permite tocar el material. Las principales fortalezas observadas en la actividad son una buena planeación, buena conciencia de las necesidades estudiantiles y la habilidad para mantener su atención. Las dos principales dificultades observadas son que se requiere una selección de cuentos más adecuada y que tengan mensajes positivos y significativos, y mayor dinamismo al realizar la actividad, ya que en ciertos momentos suena repetitiva.

En cuanto a la relación del resto de estudiantes con la niña con NEE, se observa aceptación, afectividad y respeto, y en cuanto a la maestra y los niños, se observa buena aceptación, afectividad y respeto mutuo.

En general, la actividad realizada es apropiada, apoyada con un material vistoso y que despierta el interés de los niños y las niñas, tomando en cuenta los diferentes estilos y ritmos 
doi: http://dx.doi.org/10.15359/ree.20-1.1

URL: http://www.una.ac.cr/educare

CORREO: educare@una.cr

de aprendizaje, y dando cierta libertad de exploración sobre todo a la niña con NEE; por último, cabe mencionar que la actividad es congruente con los aprendizajes y lineamientos esperados para el nivel de preescolar.

\section{Resultados de aprovechamiento y nivel cognitivo}

A continuación se muestran en la figura 1 los promedios por grupo en habilidades cognitivas, se trata de los puntajes escalares. Como se observa en esta gráfica, los grupos de las ERI, tienen mejores puntajes en promedio en todas las áreas evaluadas que sus pares de CAM (línea punteada). La mayor diferencia se presenta en las tareas de palabras incompletas, integración visual y memoria para frases.

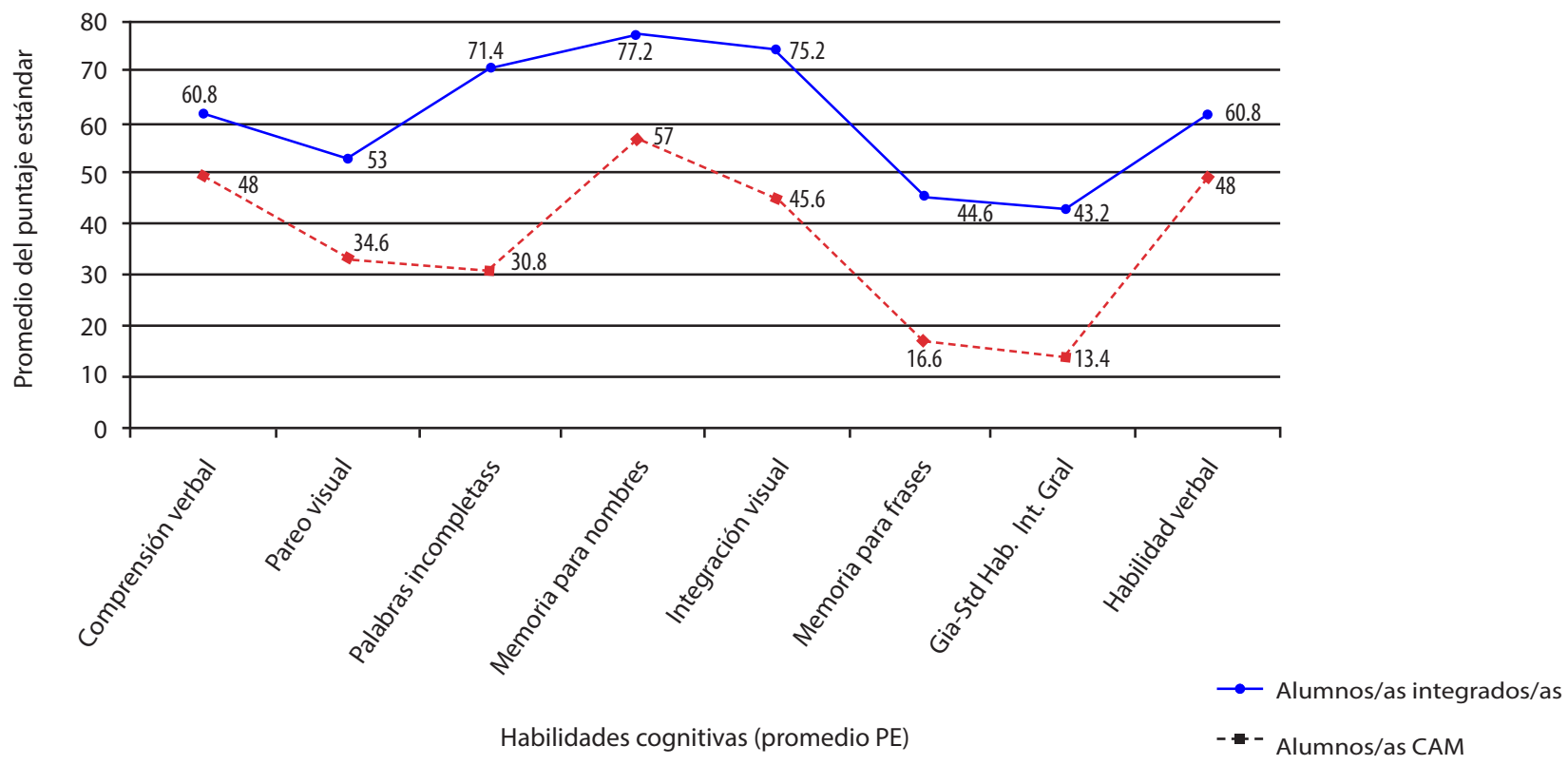

Figura 1. Puntajes del área cognitiva de la Batería III Woodcock-Muñoz por estudiantes del CAM y las alumnas y alumnos integrados. Nota. Elaboración propia.

En la figura 2 se muestran los puntajes escalares promedio del área de aprovechamiento escolar; nuevamente los niños y las niñas de las ERI (línea continua) muestran un mejor desempeño promedio que sus pares de CAM, las mayores diferencias se observan en ortografía y vocabulario sobre dibujos. 
doi: http://dx.doi.org/10.15359/ree.20-1.1

URL: http://www.una.ac.cr/educare

CORREO: educare@una.cr

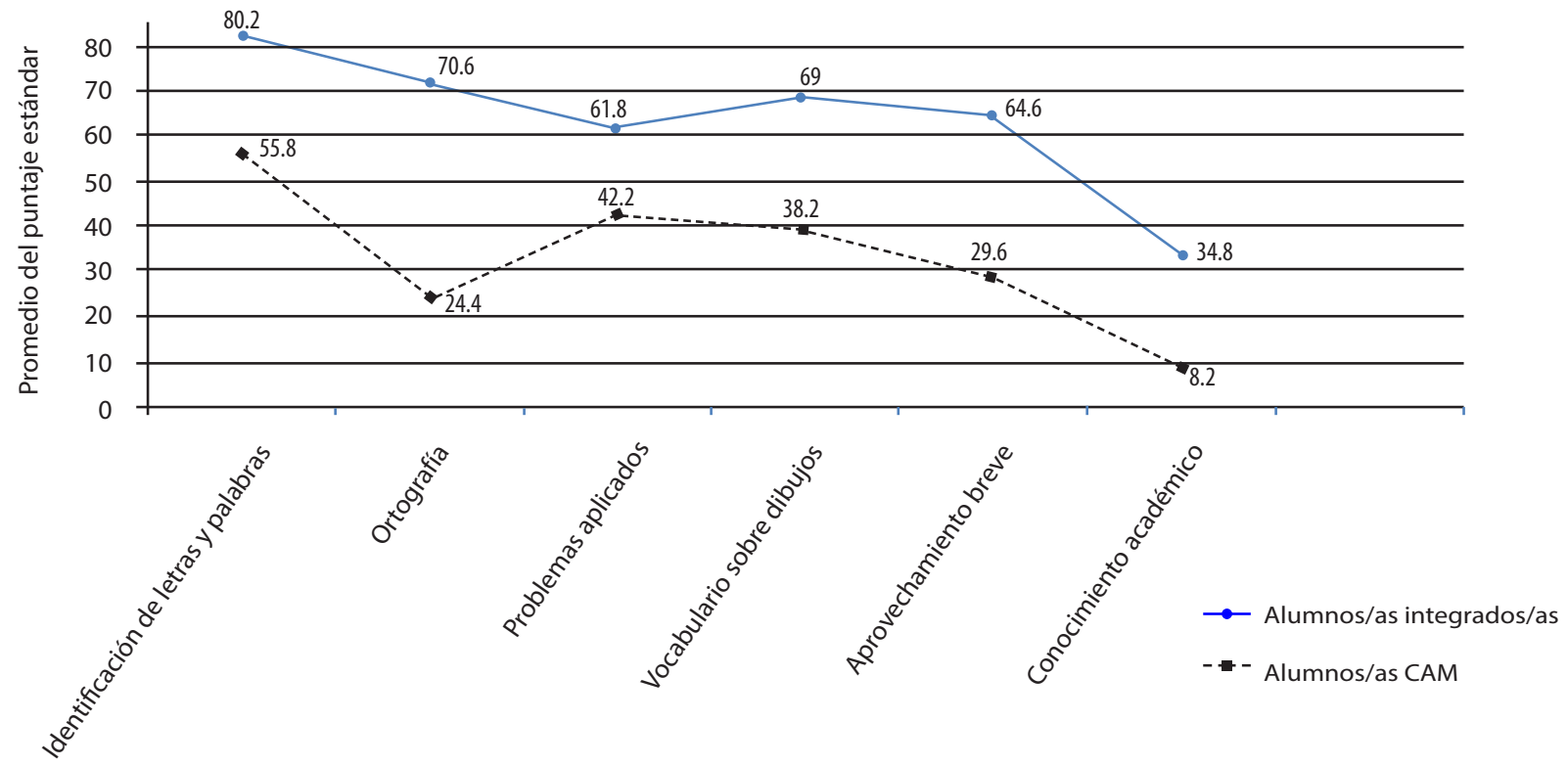

Aprovechamiento académico (promedio PE)

Figura 2. Puntajes del área de aprovechamiento de la Batería III Woodcock-Muñoz por estudiantes del CAM y las alumnas y los alumnos integrados. Nota: elaboración propia.

\section{Discusión y conclusiones}

Una consideración importante en relación con el objetivo general es que, aunque hay diferencias en cuanto a habilidades cognitivas y aprovechamiento escolar de los niños y niñas con discapacidad que asisten al Centro de Atención Múltiple (CAM) o a la escuela regular integradora (EWRI), los puntajes de ambos grupos se ubican a más de dos desviaciones estándar de la media (100). Los niños y niñas que asisten a la ERI obtienen puntajes ubicados a menos de dos desviaciones estándar solamente en tres pruebas de habilidad cognitiva, mientras que quienes asisten a CAM en ninguna. En rendimiento, ERI obtiene puntajes ubicados a menos de dos desviaciones estándar en dos pruebas, CAM en ninguna. En otras palabras, los alumnos y alumnas de esta investigación tienen habilidades cognitivas y un rendimiento escolar muy pobres (aunque ERI obtiene puntajes menos bajos). Esta situación debe tenerse presente en los siguientes comentarios de este apartado. 
doi: http://dx.doi.org/10.15359/ree.20-1.1

URL: http://www.una.ac.cr/educare

CORREO: educare@una.cr

Los alumnos y alumnas que asisten a la escuela regular obtienen un mayor puntaje en el compuesto habilidad intelectual general; en otras palabras, parecen contar con un poco más de recursos cognitivos que los alumnos y alumnas de CAM (Mather y Woodcock, 2005). En cambio, en el compuesto de habilidad verbal la diferencia es menos notoria, a pesar de que la prueba en la que se aprecian más diferencias es la de palabras incompletas. Por otro lado, el rendimiento escolar de quienes asisten a la ERI (compuestos, aprovechamiento breve y conocimiento académico) es muy superior al de los alumnos y alumnas de CAM, lo cual puede deberse al efecto de la convivencia con otros compañeros y compañeras, es decir, el alumnado con discapacidad participa de un ambiente más estimulante y retador. Otro factor que puede influir en estos resultados en el estilo más centrado en el aprendizaje escolar evidenciado en la escuela regular, aunque seguramente las diferencias socioculturales tienen una influencia considerable.

El análisis de los datos muestra que entre los dos grupos estudiados hay diferencias importantes en cuanto a edad, nivel sociocultural (educación de progenitores, condiciones de vida, acceso a material didáctico y tecnológico), estilo de crianza y rutinas familiares, lo que, en general, pone al alumnado de la escuela especial (CAM) en desventaja en relación con el que asiste a la escuela regular. Estos resultados y el hecho de no contar con información acerca de la integración educativa sugieren que las familias de niños que asisten al CAM tienen menos recursos para tomar una decisión informada con respecto al tipo de institución al que pueden inscribir a sus hijos e hijas.

Debe tomarse en cuenta que, en general, progenitores de los niños y niñas con NEE asociadas a una discapacidad enfrentan estresores muy importantes, pues:

a) Las necesidades educativas especiales que presentan sus hijos e hijas a veces requieren apoyos muy amplios, que el sistema educativo mexicano no proporciona, a pesar de lo que menciona la Ley de Inclusión de Personas con Discapacidad y la Reglamentación de la misma ley (Estados Unidos Mexicanos. Cámara de diputados del H. Congreso de la Unión, 2011, 2012). Por ejemplo, en cuanto a apoyos personales, es responsabilidad casi exclusiva de sus progenitores conseguir sillas de ruedas o prótesis auditivas, por mencionar un par de ejemplos. Muchas familias no saben gestionar estos recursos en las instancias apropiadas, y la mayoría de estos niños y niñas se las tiene que arreglar sin estos apoyos.

b) Los niños y niñas con NEE enferman con cierta frecuencia, lo cual incrementa el ausentismo escolar y los gastos de las familias.

c) La sociedad mexicana sigue siendo profundamente discriminadora; una de las poblaciones más discriminadas es la de las personas con discapacidad (Consejo nacional para el desarrollo y la inclusión de las personas con discapacidad, 2012). 
doi: http://dx.doi.org/10.15359/ree.20-1.1

URL: http://www.una.ac.cr/educare

CORREO: educare@una.cr

Mientras que cuatro de los niños de CAM tienen siete años (uno tiene seis), los de las ERI tienen entre 5 y 6 años, a pesar de cursar el mismo grado escolar. Es una práctica común en los CAM la permanencia prolongada en cada uno de los niveles educativos. Los niños y las niñas permanecen más tiempo en preescolar y más tiempo en primaria, lo cual hace muy difícil igualar muestras para la realización de investigaciones.

En las familias de las ERI se observa una mayor cantidad de recursos económicos (por ejemplo, se trasladan en auto propio) y para el aprendizaje (la escolaridad de los progenitores es de secundaria o más, en la mayoría de las casas hay computadora) que en las familias de los niños de CAM, cuyos padres, único sustento de la familia, tienen empleos de calificación intermedia y en sus casas hay pocos recursos para el aprendizaje (un limitado número de libros y en ningún caso hay computadora), tienen que trasladarse en transporte público y tienen jornadas largas para acceder a la escuela.

Los niños y niñas de CAM utilizan mucho tiempo en transportarse, pues viven lejos de la escuela (hay pocos CAM en comparación con el número de escuelas regulares) y hacen uso del transporte público (que no es muy eficiente); muestran una mayor exposición al televisor (cuatro horas diarias en promedio) y menor variedad de actividades recreativas que sus pares de escuela regular, en comparación con sus pares de las ERI, quienes ven menos televisión (entre una y dos horas diarias) y realizan actividades fuera del hogar en forma cotidiana (visitar familiares, ir al parque, etc.).

Lo anterior parece confluir en la misma situación mencionada previamente: los progenitores de los niños y niñas que asisten al CAM de la muestra tienen menos recursos económicos, educativos, culturales y sociales que la muestra de los progenitores de niños que asisten a las ERI. Asimismo, tienen menos expectativas sobre su futuro educativo.

Llama la atención que, en general, las madres están satisfechas con la educación y el apoyo psicoeducativo que reciben sus hijos e hijas. Es probable que esta buena opinión por parte de las mamás de quienes asisten al CAM se vea influida por su intensa participación en las actividades de la escuela, pues, debido a que regresar a sus casas al llevar a sus hijos al CAM les toma mucho tiempo y dinero, muchas de ellas se quedan la jornada completa en la institución, apoyando al personal docente.

Las observaciones realizadas muestran algunas diferencias en el funcionamiento de las escuelas. Como se mencionó, en el CAM hay una participación activa de las madres de familia, quienes apoyan al personal docente durante el tiempo de clase. Las condiciones físicas del CAM son de cierto deterioro y desorden, frente a la ERI que cuenta con más recursos didácticos y de organización. La clase observada en el CAM está centrada en habilidades específicas que resultan poco interesantes para los alumnos y alumnas, por lo que rápidamente pierden el interés; en contraste, la clase observada en la ERI logra captar la atención y promueve la 
participación activa. Aunque se trata de una sola observación en cada contexto, llama la atención la diferencia de estilo pedagógico, manejo del tiempo, ambiente de aula y en los aprendizajes esperados de parte del alumnado. Mientras que en el CAM hay un énfasis por "resolver el problema de los alumnos" mediante la ejercitación de la expresión oral y la relajación de músculos faciales, presumiblemente responsables de sus dificultades expresivas, en la ERI el énfasis está puesto en el aprendizaje a través de recursos interesantes para el alumnado (lectura de un cuento). Es probable entonces que en la docente de CAM haya expectativas muy bajas con respecto al aprendizaje de sus alumnos y alumnas, situación encontrada en la mayoría de las investigaciones sobre el tema, por ejemplo, en la realizada por la Red internacional de investigadores y participantes sobre integración educativa (2006).

Dado el reducido tamaño de la muestra y el hecho de que no se pudieron controlar mejor las variables socioculturales, resulta difícil llegar a una conclusión sobre la influencia del tipo de escuela sobre el rendimiento escolar de sus estudiantes, sobre todo porque también se relaciona con factores socioculturales e incluso con condiciones escolares y áulicas. Por tanto, esta investigación no puede ser concluyente, pero sí sugiere algunas ventajas de la escuela regular frente a la escuela especial. La experiencia de quienes suscriben este artículo durante los primeros años en que se inició el proceso de integración educativa en el país también va en ese sentido, pues fueron testigos de cómo los niños y niñas que habían permanecido en sus casas o pasaron de educación especial a educación regular experimentaron cambios a veces espectaculares en su socialización y sus aprendizajes (García et al., 2003). Las ventajas observadas en la presente investigación, sin embargo, son relativas, pues las habilidades cognitivas y el rendimiento académico del alumnado son muy pobres.

Es necesario realizar investigaciones con mayor número de casos, mejores controles en las variables y un seguimiento sistemático de la forma en la que se realiza la enseñanza en cada uno de estos ambientes escolares para comprender mejor las condiciones en las que se realiza la educación y los beneficios que se ofrece al estudiantado, a fin de sugerir recomendaciones para mejorar la calidad de la educación que reciben los alumnos y alumnas con discapacidad o $\mathrm{NEE}$, independientemente del tipo de centro educativo al que asistan.

A pesar de la gran complejidad que representa su diseño, investigaciones como la presente son imprescindibles para darles visibilidad a los CAM, pues, como comenta la Red internacional de investigadores y participantes sobre de la integración educativa (2006), han permanecido a la zaga en el sistema educativo mexicano. Además, las investigaciones son urgentes si queremos evitar que los niños y niñas con necesidades educativas especiales asociadas con discapacidad o sin esta se conviertan en personas adultas estigmatizadas, imposibilitadas de ejercer plenamente sus derechos (Consejo nacional para prevenir la discriminación [CONAPRED], s. f.), y si queremos que toda la población aprenda a vivir con sus semejantes, sin importar sus diferencias. 
doi: http://dx.doi.org/10.15359/ree.20-1.1

URL: http://www.una.ac.cr/educare

CORREO: educare@una.cr

\section{Agradecimientos}

Los autores agradecen la colaboración del Lic. Mario Geraldo López Quirino, por su colaboración en la realización del presente trabajo.

\section{Referencias}

Blanco, R., Mascardi, L. y Narvarte, L. (2010). Sistema regional de información educativa de los estudiantes con discapacidad SIRIED. Propuesta metodológica. Santiago de Chile: Unesco. Recuperado de http://unesdoc.unesco.org/images/0019/001909/190974s.pdf

Brunner, J. J. y Elaqcua, G. (2004). Factores que inciden en una educacion efectiva. Evidencia internacional. La educación. Revista Interamericana de Desarrollo Educativo, 48-49(139140), 1-11. Recuperado de http://ww2.educarchile.cl/UserFiles/P0001/File/Factores\%20 q\%20inciden\%20en\%20una\%20educacion\%20efectiva 2004.pdf

Carrasco, G. (2008). Influencia del capital cultural, capital económico y capital social basado en la familia sobre el rendimiento de los estudiantes. Un análisis comparativo. Lima: CIES. Recuperado de http://old.cies.org.pe/files/documents/investigaciones/educacion/ influencia-del-capital-cultural-economico-y-capital-social-basado-en-la-familia-sobre-elrendimiento-de-los-estudiantes-un-analisis-comparati.pdf

Cervini, R. (Setiembre, 2002). Desigualdades en el logro académico y reproducción cultural en Argentina. Revista Mexicana de Investigación Educativa, 7(16), 445-500. Recuperado de http://www.redalyc.org/pdf/140/14001604.pdf

Consejo nacional para el desarrollo y la inclusión de las personas con discapacidad. (2012). Encuesta nacional sobre discriminación en México. Enadis 2010. Resultados sobre personas con discapacidad. México: Autor. Recuperado de http://www.conapred.org.mx/userfiles/ files/Enadis-PCD-Accss.pdf

Consejo nacional para prevenir la discriminación (CONAPRED). (s. f.). Discriminación personas con discapacidad. Recuperado de http://www.conapred.org.mx/index. php? contenido $=$ pagina\&id $=133 \&$ id opcion $=46 \& o p=46$

Dyson, A., Farrell, P., Polat, F., Hutcheson, G. y Gallannaugh, F. (2004). Inclusion and pupil achievement. Nottingham: University of Newcastle. Recuperado de http://webarchive. nationalarchives.gov.uk/20130401151715/http://www.education.gov.uk/publications/ eorderingdownload/rr578.pdf 
doi: http://dx.doi.org/10.15359/ree.20-1.1

URL: http://www.una.ac.cr/educare

CORREO: educare@una.cr

Estados Unidos Mexicanos. Cámara de diputados del H. Congreso de la Unión. (2011). Ley general para la inclusión de las personas con discapacidad. Recuperado de http://www. educacionespecial.sep.gob.mx/pdf/doctos/1Legislativos/5Ley General Inclusion de Personas Discapacidad.pdf

Estados Unidos Mexicanos. Cámara de diputados del H. Congreso de la Unión. (2012). Reglamento de la ley general para la inclusión de las personas con discapacidad. Recuperado de http:// normatecainterna.sep.gob.mx/work/models/normateca/Resource/229/1/images/ reglamento ley general inclusion personas discapacidad.pdf

Estados Unidos Mexicanos. Cámara de diputados del H. Congreso de la Unión. (2015). Ley general de educación. Recuperado de https://www.sep.gob.mx/work/models/sep1/ Resource/558c2c24-0b12-4676-ad90-8ab78086b184/ley general educacion.pdf

Ezcurra, M. E. (2004). La calidad de la atención educativa de los niños y jóvenes con discapacidad en los centros de atención múltiple (Informe final de investigación). Recuperado de: http:// www.cee.iteso.mx/BE/25/371.91\%20EZCi\%2018EzcurraMarta.pdf

García, I., Escalante, I., Escandón, M., Fernández, L. G., Mustri, A. y Puga, I. (2003). Proyecto de investigación e innovación integración educativa. Informe parcial de investigación (Ciclos escolares 1997-1998). En Integración educativa. 1996-2002. (Informe final, disco compacto). México-España: SEP, Subsecretaría de Educación Básica y Normal, Fondo Mixto de Cooperación Técnica y Científica y Cooperación Española.

García, I., Romero, S., Motilla, K. y Zapata, C. I. (2009). La reforma fallida de los centros de atención múltiple en México. Actualidades Investigativas en Educación, 9(2), 1-21. Recuperado de http://revista.inie.ucr.ac.cr/index.php/aie/article/viewFile/325/324

Gil, J. (Setiembre-diciembre, 2013). Medición del nivel socioeconómico familiar en el alumnado de educación primaria. Revista de Educación, 362. doi: 10-4438/1988-592X-RE-2011-362-162

Gobierno Federal. Comisión de política gubernamental en materia de derechos humanos (s. f.). Glosario de términos sobre discapacidad. Programa de fortalecimiento. Educación especial integración educativa. Recuperado de http://www.semar.gob.mx/derechos humanos/ glosario terminos discapacidad.pdf

Guajardo, E. (2010). La desprofesionalización docente en educación especial. Revista Latinoamericana de Inclusión Educativa, 4(1), 105-126. Recuperado de http://www.rinace. net/rlei/numeros/vol4-num1/art5.pdf

Gutiérrez, S. y Montañez, G. S. (Julio-diciembre, 2012). Análisis teórico sobre el concepto de rendimiento escolar y la influencia de factores socioculturales. Revista Iberoamericana para la Investigación y el Desarrollo Educativo, 9, 1-21 
doi: http://dx.doi.org/10.15359/ree.20-1.1

URL: http://www.una.ac.cr/educare

CORREO: educare@una.cr

Hernández, E. y González, M. J. (2011). Modelo de ecuación estructural que evalúa las relaciones entre el estatus cultural y económico del estudiante y el logro educativo. Revista Electrónica de Investigación Educativa, 13(2), 188-203. Recuperado de http://redie.uabc.mx/redie/ article/view/290/454

Jiménez, C. (1988). Condición socioeconómica de la familia y rendimientos escolares de los hijos al término de la E.G.B. Revista de Educación, 287, 55-70. Recuperado de $\quad$ http://www.mecd.gob.es/dctm/revista-de-educacion/articulosre287/re28703. pdf?documentld=0901e72b813c2ffg

López, F. X. (s. f.) Consideraciones en torno al proceso de atención de los menores con trastornos profundos del desarrollo en el Centro de Atención Múltiple de Educación Especial. Recuperado de http://www.quadernsdigitals.net/datos/hemeroteca/r 24/ nr 280/a 3630/3630.htm

Lupton, R., Thrupp, M. y Brown, C. (2010). Special educational needs: A contextualized perspective [Necesidades educativas especiales: Una perspectiva contextualizada]. British Journal of Educational Studies, 58(3), 267-284. doi: 10.1080/00071005.2010.500607

Mather, N. y Woodcock, R. (2005). Woodcock-Johnson III pruebas de aprovechamiento. Manual del examinador (Trad. L. Wolfson). Itasca, IL: Riverside Publishing.

OREALC/Unesco (2008). Los aprendizajes de los estudiantes de América Latina y el Caribe. Segundo estudio regional comparativo y explicativo. Santiago: Autor.

Organización para la Cooperación y el Desarrollo Económico (OECD). (2010). Pisa 2009 Results: What students know and can do. Student performance in reading, matematics and science [Lo que los estudiantes saben y pueden hacer. Rendimiento de los estudiantes en lectura, matemáticas y ciencia] (Vol. I). Recuperado de http://www.oecd.org/pisa/ pisaproducts/48852548.pdf

Peña, C. M. (2002). Rendimiento escolar en Chile en establecimientos públicos y privados: ¿Qué nos muestra la nueva evidencia? (Tesis de maestría). Universidad de Chile. Recuperado de http://www.opech.cl/bibliografico/Doc Financiamiento/ RendimientoEscolarEnChileEnEstablecimientosPublicosYPrivadosPena.pdf

Poder Ejecutivo Federal. (19 de mayo de 1992). Acuerdo nacional para la modernización de la educación básica. Diario Oficial. Recuperado de http://www.sep.gob.mx/work/models/ sep1/Resource/b490561c-5c33-4254-ad1c-aad33765928a/07104.pdf 
doi: http://dx.doi.org/10.15359/ree.20-1.1

URL: http://www.una.ac.cr/educare

CORREO: educare@una.cr

Ponce, V. M., Pérez, V., López, L. M. y Hernández, A. C., (2006). Políticas y significados educativos de los agentes de los centros de atención múltiple que ofrecen educación primaria en el Estado de Jalisco (Proyecto SEP-SEByN-CONACYT-2003-CO-47/A-1). Guadalajara: Gobierno de Jalisco. Poder Ejecutivo. Secretaría de Educación. Recuperado de http://portalsej.jalisco. gob.mx/sites/portalsej.jalisco.gob.mx.investigacion-educativa/files/pdf/informe.pdf

Red internacional de investigadores y participantes sobre integración educativa. (2006). Evaluación externa del programa nacional de Fortalecimiento de la educación especial y de la integración educativa (Informe final 2005). México: Subsecretaría de Educación Básica y Normal. Recuperado de http://www.educacionespecial.sep.gob.mx/coneval/eval/eval anteriores/evaluacion05.pdf\#page $=5 \& z 00 m=$ auto, $-130,42$

Ruijs, N. M.; van der Veen, I. y Peetsma, T. D. (2010). Inclusive education and students without special educational needs [Educación inclusiva y estudiantes sin necesidades educativas especiales]. Educational Research, 52(4), 351-390. doi: 10.1080/00131881.2010.524749

Samaniego, P. (2009). Capítulo VI. Hacia una educación inclusiva. En P. Samaniego (Directora). Personas con discapacidad y acceso a servicios educativos en Latinoamérica. Breve análisis de situación (pp. 323-436). Quito: Ediciones Simca. Recuperado de http://www.cermi.es/ es-ES/ColeccionesCermi/Cermi.es/Lists/Coleccion/Attachments/57/cermies391vol.pdf

Schrank, F. A., McGrew, K. S., Ruef, M. L., Alvarado, C. G., Muñoz-Sandoval, A. F. y Woodcock, R. W. (2005). Overview and Technical Supplement (Bateria III Woodcock-Muñoz Assessment Sevice Bulletin, No. 1). [Breve reseña y suplemento técnico. Batería III Woodcock-Muñoz]. Itasca, IL: Riverside Publishing.

Secretaría de Educación Pública (SEP). (2002). Programa nacional de fortalecimiento de la educación especial y de la integración educativa. México: Autor. Recuprado de http://www. educacionespecial.sep.gob.mx/pdf/publicaciones/ProgNal.pdf

Secretaría de Educación Pública (SEP) (2006). Orientaciones generales para el funcionamiento de los servicios de educación especial. México: Autor. Recuperado de http://www. educacionespecial.sep.gob.mx/pdf/publicaciones/libromorado.pdf

Secretaría de Educación Pública (SEP). (2012). Programa de fortalecimiento de la educación especial y de la integración educativa [Diapositivas en PowerPoint] Recuperado de http:// www.educacionespecial.sep.gob.mx/pdf/tabinicio/2012/Datos pfeeie 2012.pdf

Secretaría de Educación Pública (SEP). (2013). Estadísticas históricas 1983-2012. México: Autor. Recuperado de http://www.snie.sep.gob.mx/estadisticas educativas.html

Sentín, D. (2001). Influencia de los factores socieconómicos en el rendimiento escolar internacional: hacia la igualdad de oportunidades educativas. Documento de trabajo o Informe técnico, 1, 1-27. Recuperado de http://eprints.ucm.es/6725/1/0101.pdf 
doi: http://dx.doi.org/10.15359/ree.20-1.1

URL: http://www.una.ac.cr/educare

CORREO: educare@una.cr

Tomasevsky, K. (2002). Contenido y vigencia del derecho a la educación (Serie Cuadernos Pedagógicos). San José de Costa Rica: Instituto Interamericano de Derechos Humanos. Recuperado de http://cedh-chiapas.org/documentos-dh/Instituto\%20 Interamericano\%20de\%20Derechos\%20Humanos \%20-\%20Publicaciones/ Colecci\%C3\%B3n\%20Cuadernos\%20Pedag\%C3\%B3gicos.\%20Contenido\%20y\%20 vigencia\%20del\%20derechos\%20a\%20la\%20educaci\%C3\%B3n.PDF

Woodcock, R. y Muñoz-Sandoval, A. F. (1996). Batería Woodcock-Muñoz: Pruebas de habilidad cognitiva-revisada. Itasca, IL, EUA: Riverside Publishing Company.

\section{Cómo citar este artículo en APA:}

García-Cedillo, I. y Romero-Contreras, S. (Enero-abril, 2016). Influencia del tipo de escuela (especial o regular) y algunas variables socioeconómicas sobre el aprovechamiento escolar del alumnado con discapacidad. Revista Electrónica Educare, 20(1), 1-26. doi: http://dx.doi.org/10.15359/ree.20-1.1

Nota: Para citar este artículo en otros sistemas puede consultar el hipervínculo "Como citar el artículo" en la barra derecha de nuestro sitio web: http://www.revistas.una.ac.cr/index.php/EDUCARE/index 